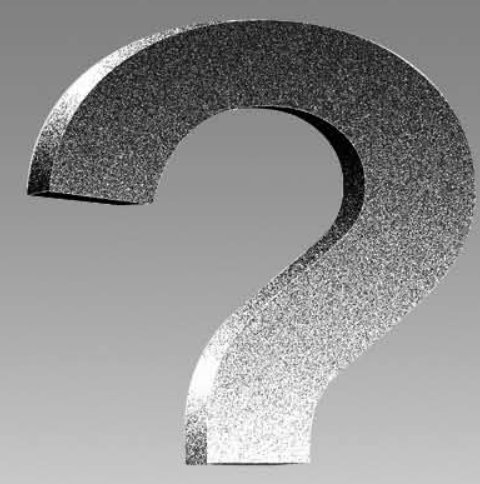

\title{
La confianza y la reputación como variables del desarrollo económico y la imagen país
}

Reputation and Trust as Variables of Economic Development and Nation Branding

Claudia Labarca, PhD Program, Universidad de Durham, Reino Unido. [claudia_labarcalahotmail.com]

Recibido: 24/10/2008/Aceptado: $20 / 11 / 2008$

I Este artículo propone la necesidad de incorporar las llamadas ¿ «variables blandas» al análisis sobre desarrollo económico,

E centrándose específicamente en conceptos como reputación y

ज confianza. Para ello, se revisan enfoques teóricos tales como

$\approx$ Poder Blando, Diplomacia Pública y la reciente teoría de Identidad Competitiva o Nation Branding. El artículo también examina el caso de Chile por tratarse de un país abierto comercialmente al mundo $y$, por ende, sujeto a la competitividad del mercado internacional no sólo en la oferta de sus productos y servicios, sino también en la atracción de inversión extranjera y turismo. Es en este contexto de un Chile globalizado e inserto en la actividad económica mundial donde variables como reputación y confianza aparecen como claves para su desarrollo.

Palabras claves: Desarrollo económico, confianza, reputación, Chile,

Imagen país.
1 This article suggests the need to incorporate so-called «soft variables» into $\underset{\sigma}{ \pm}$ the analysis of economic development with a specific focus on concepts such \pm as reputation and trust and reviews theoretical positions such as Soft Power I Public Diplomacy, and the recent theory of Competitive Identity or Nation $\varangle$ Branding. The article also examines the case of Chile as a commercially open country that is subject to international competition with respect to its products, services, tourism, and in attracting foreign investment. It is within this context of a globalized Chile integrated into the world economic activity that variables such as reputation and trust appear as keys for its development.

Keywords: Economic development, trust, reputation, Chile, Nation Branding. 
D rante los siglos XIX y XX, el predominio de la ilustración europea, unido a la teoría de elección racional y el neoclasicismo (Granovetter, 1985a; Schech \& Haggis, 2000; Throsby, 2001), ha centrado el debate sobre el desarrollo económico fundamentalmente en determinar si es el mercado o el Estado el mecanismo más apropiado para la generación de riqueza en las naciones (Gilpin \& Gilpin, 2001), dejando fuera del análisis aspectos socioculturales. En los noventa este debate se enmarcó en la discusión sobre el fenómeno de la globalización y la puesta en duda de la continuidad del Estado Nación como unidad básica del escenario internacional, frente a la creciente importancia de las compañías multinacionales (Friedman, 2006; Ohmae, 1996).

Sin embargo, más allá del debate sobre las consecuencias de la globalización para el futuro del Estado (que inundó la literatura durante los años noventa), actualmente existe cierto consenso en algunas ideas básicas compartidas tanto por la academia, como por quienes definen las políticas públicas en la mayor parte del mundo. Por un lado, se reconoce la existencia de nuevos actores (como las corporaciones o las ONG) en la dinámica de poder de las relaciones internacionales, (Bhagwati, 2004; Held, 1999; J. Nye, 1990), y por otro, se entiende el papel que han ejercido los procesos de globalización en la redefinición del concepto de Estado: «El Estado ha muerto, larga vida al Estado» (Dicken, 2003, pp.122) $)^{1}$.

Particularmente desde la perspectiva económica, hay acuerdo en determinar que el rol del Estado debe consistir, al menos, en generar las condiciones necesarias (marco legal, estabilidad institucional y macroeconómica, entre otras variables) para que el mundo privado pueda desarrollarse exitosamente.

En las últimas décadas, sin embargo, el análisis sobre desarrollo económico -y por ende la manera más adecuada de alcanzarlo- ha comenzado a dar cabida a variables socioculturales antes ignoradas o «soft variables» (Sztompka, 1999), lo que se ha traducido al español como «variables blandas». El fracaso del llamado Consenso de Washington ${ }^{2}$ en muchos de los países latinoamericanos ha ayudado a evidenciar la necesidad de entender el problema desde una perspectiva más amplia e interdisciplinaria.

Así han surgido con fuerza teorías desde la sociolo- gía, antropología e incluso el marketing y las comunicaciones que reivindican el rol de aspectos socioculturales en el desarrollo económico, siendo las más reputadas las teorías de Capital Social (Bourdieu, 2005; Coleman 1988; Dasgupta \& Serageldin, 2000; Fukuyama, 1995; Putnam, 1993, 2000, 2007), cuyo eje central es la existencia de redes sociales basadas en la confianza y el papel que estas tienen en el crecimiento.

Este artículo pretende analizar e interrelacionar los conceptos de confianza, reputación y comunicación. De este modo, se plantearán los fundamentos teóricos -basados en los enfoques de la sociología, relaciones internacionales, marketing y comunicaciones- en relación a la importancia que estas «soft variables» tienen para el desarrollo económico. Esto es particularmente relevante en el caso de Chile, pues, como economía de mercado abierta al exterior (cuenta con una red de 57 acuerdos comerciales con 19 países del mundo, lo que redunda en una liberalización de tarifas con el 86\% del PIB mundial $^{3}$ ), debe posicionarse competitivamente en el mercado internacional ${ }^{4}$.

Es necesario aclarar que existen otras variables igualmente relevantes para la construcción de confianza, pero que por razones de espacio no es posible analizar aquí, tales como la importancia de la interacción (Dasgupta \& Serageldin, 2000; Gambetta, 1988), el interés encapsulado (Hardin, 2002), la confianza como expectativas de comportamiento (Knight, 2001), la confianza basada en identidades y características adscritas (Fukuyama, 1995), la cercanía social (Glaeser, 2002a), las normas culturales compartidas (Granovetter, 1985b; Knight, 2001) y el marco institucional (Sen, 1999; Zucker, 1986), entre otras.

\section{CONFIANZA: LA «VARIABLE PERDIDA» EN EL DESARROLLO ECONÓMICO}

En la década de los noventa, el escritor, académico y asesor de la Casa Blanca Francis Fukuyama analizó desde la perspectiva de la confianza el desarrollo económico de Estados Unidos y algunas sociedades europeas y asiáticas en su libro Confianza: Virtudes Sociales y Creación de Prosperidad (Fukuyama, 1995), concluyendo que determinados factores culturales inciden en los grados de confianza generalizada de una sociedad, lo que a su vez, redunda en el desarrollo económico, básicamen-
1 Dicken expone que las principales tareas del Estado en un contexto de globalización son: actuar como «contenedor» (container) de estructuras culturales, prácticas e instituciones, así como también dictar las políticas que regulan el comercio, la inversión extranjera y la industria.

2 El Consenso de

Washington es el nombre dado a la política exterior impulsada principalmente por los Estados Unidos a través del Banco Mundial y del Fondo Monetario Internacional que, en la década de los ochenta y parte de los noventa, consistió en una serie de préstamos asociados a determinadas condiciones impuestas por estos entes financieros. Una de las críticas hechas a este modelo radica en la imposibilidad de aplicar una idea sin consideraciones de tipo socioculturales. Para un agudo análisis sobre el llamado Consenso de Washington en América Latina, ver Rodrik, After Liberalism, what? (Rodrik, 2002)

3 De acuerdo al CIA Factbook (https://www.cia. gov/library/publications/ the-world-factbook/geos/ ci.htm (\#Econ), Chile es el país con mayor número de acuerdos comerciales en el mundo. 
4 Según estadísticas de ProChile, en el año 2007 se exportaron US\$68.295.7 millones FOB, esto es US\$10.179,2 millones más que en 2006 (Guardia, 2008)

Sin embargo, el 55,9\% correspondió a cobre. Esta composición de la canasta exportadora chilena

con fuerte acento en el commodity cobre, resalta aún más la necesidad de diversificación y, por ende,

la importancia de gestionar la reputación y confianza de Chile en el escenario internacional, de manera de incentivar el desarrollo de un nuevo y más variado portafolio de productosy servicios, además de atraer

la inversión extranjera.

5 En Economía, se conoce como costos de transacción a todos aquellos costos que van unidos a un proceso de intercambio económico, tales como comisiones y búsqueda de información, así como también los procesos informales asociados.

6 Sus detractore argumentan que, desde este punto de partida, habrían sociedades que estarían condenadas al subdesarrollo mientras que otras estarían destinadas a la prosperidad (Said, 2002). Si a ello se suma la dificultad de medir conceptos culturales y

el disenso que existe en

la propia definición de cultura -ya en 1952, los investigadores Kroeber and Kluckon compilaron más de 164 definiciones de cultura (Thomas, 1999)-, la teoría pierde fuerza a medida que se profundiza en su análisis. te afectando los llamados costos de transacción ${ }^{5}$. Desde esta perspectiva -weberiana en su esencia y culturalista en la práctica- determinadas sociedades poseerían en sí mismas las condiciones necesarias para crear prosperidad, mientras que en otras, factores culturales (inherentes a la identidad cultural de las naciones) serían constrictores del desarrollo económico. Esta tendencia «culturalista» es también compartida por renombrados académicos (Harrison, 1992; Huntington \& Harrison, 2000) que, si bien tienen el mérito de poner en el debate el tema de factores culturales como variables que inciden en el desarrollo económico, han sido blanco de fuertes críticas, principalmente por su visión estática de la cultura y por su metodología ${ }^{6}$. Desde Fukuyama, mucho se ha escrito sobre el tema de la confianza en sus niveles micro (confianza interpersonal), mezo (confianza entre compañías, asociaciones y agrupaciones) y macro (confianza generalizada)

En este último nivel, abundante literatura ha argumentado que los países con altos niveles de confianza generalizada son los más propensos al desarrollo económico. El concepto de confianza está emergiendo como el «factor perdido» (Humpfrey, 1998) que explicaría por qué unos países se desarrollan más rápido que el resto. En la conocida encuesta mundial de valores (Inglehart, 2004) países como Noruega, Suiza o Suecia presentan altos niveles de confianza, en comparación con algunos países africanos por ejemplo, cuyos bajos niveles de confianza serían una de las explicaciones para su pobre desempeño económico, existiendo así una correlación empírica entre las variables de confianza y desarrollo (Knack \& Zak, 2001).

Sin embargo, en un mundo eminentemente globalizado y abierto comercialmente, poca literatura ha fijado su atención en los procesos de confianza que se construyen entre las naciones y cómo diversos elementos, entre ellos los ligados a la comunicación, inciden en la construcción de esta confianza ${ }^{7}$. Por eso, ésta debe ser entendida desde distintos niveles, vale decir desde el ámbito diplomático (esto es una comunicación desde un Estado a otro) hasta la comunicación que se produce entre los actores privados de diferentes naciones y la sinergia e interacción entre ellos.

\section{ENLACE ENTRE CONFIANZA Y COMUNICACION}

Parte importante de la literatura existente en esta área, explica la directa conexión entre el desarrollo de confianza entre dos actores (sean éstos personas, compañías o naciones) y la comunicación como variable independiente: «La confianza es el resultado directo de la comunicación, en donde significados compartidos se desarrollan para proveer la base necesaria que permite el comportamiento no oportunista» (Hardy, Phillips, \& Lawrence, 1998b, pp. 69). Es más, se argumenta que «la confianza es resultado de un proceso comunicativo» ya sea preexistente o creado por las partes. (Hardy, Phillips, \& Lawrence, 1998a, pp. 71). Asimismo, la literatura también ha estudiado cómo problemas de comunicación, lenguaje y entendimiento afectan negativamente los procesos de desarrollo de confianza (Child, 1998).

Desde otra perspectiva, hay acuerdo en señalar que fuente importante de desarrollo de confianza parte desde el conocimiento de la contraparte, es decir, desde la confianza basada en el conocimiento -knowledge based trust (Hardin, 2002; Knack \& Zak, 2001)-, lo que no es posible si no existe comunicación entre los actores involucrados, entendiendo a la comunicación en el amplio sentido de «dar a conocer» y también en el entendido del conocimiento que da la interacción previa (Axelrod, 1984).

Ahora bien, mucho de este conocimiento viene tamizado por la reputación que precede a la contraparte (McKnight, Harrison, \& Chervany, 2006). De acuerdo al Reputation Institute, organización que realizó un modelo sobre la importancia de la reputación en la eficacia de las empresas y donde ésta aparece estrechamente ligada a la confianza: «Las personas actúan basadas en sus sentimientos. Existe más probabilidad de que compren productos de compañías en las que ellos confíen, trabajen para organizaciones que ellos respeten y recomienden empresas que les gusten $»^{8}$ (Glaeser, 2002b). Esta afirmación es válida también en el contexto de las naciones, ya que las marcas corporativas de un país afectan directamente la reputación del territorio de origen y viceversa: «El país de origen o made in tiene un efecto significativo en las decisiones de compra de los consumidores e influye en la percepción de calidad de los productos o servicios», existiendo «una relación de mutua aportación de 
contenido entre la marca país y las principales marcas locales» (Prado, 2007).

Esta necesidad de gestionar la reputación con el objetivo de aumentar los niveles de confianza se intensifica en un mundo interconectado: «La importancia de la reputación, de hecho, tiende a incrementarse a medida que las sociedades se convierten en más grandes, más difusas y más complejas: esto se debe a que la mayoría de las transacciones humanas dependen en un cierto grado de confianza para poder proceder» (Anholt, 2008a, p. 1).

En este sentido, la confianza es uno de los factores que permiten reducir la complejidad de los sistemas (Gambetta, 1988; Luhmann, 1979) y, por ende, los niveles de riesgo. Ambas temáticas -disminución del riesgo y de la complejidad- no son temas menores en un mundo lleno de ofertas, donde el creciente número de actores (tanto privados como estatales) dificulta la toma de decisiones a la hora de preferir un producto, servicio, marca o incluso país sobre otro, ya sea en el consumo o inversión. Tal como se esbozó al inicio, los procesos de globalización han determinado que las naciones sean vistas como entes competidores en el mercado internacional (Dicken, 2003) y, por lo tanto, con la necesidad de desarrollar ventajas competitivas que le permitan posicionarse exitosamente (Porter, 2000), sea ésta real o percibida.

La perspectiva (de la competencia entre los Estados) entre policy makers y académicos ha sido fuertemente aceptada. Libros, informes gubernamentales, artículos de prensa, programas de televisión en virtualmente todos los países se adhieren al lenguaje y a la imaginería de la lucha competitiva entre los estados por una tajada mayor de la torta global (Dicken, 2003, p. 140)

Sin embargo, no sólo es evidente la urgencia de desarrollar ventajas competitivas, sino también de comunicarlas de la manera más adecuada para influenciar, persuadir y, en definitiva, generar confianza en la diversidad de públicos a los que una nación está expuesta. Desde esta perspectiva, la confianza no sólo incide en el desarrollo económico sino que también -desde la visión de las relaciones internacionales- da respuesta a las exigencias impuestas por el interés nacional.
Por lo tanto, la pregunta que cabe hacerse desde una visión teórico-práctica es determinar cuáles son las fuentes de generación de confianza y -desde la perspectiva de las políticas públicas- cuál es la mejor estrategia para lograr construirla en un escenario global competitivo, donde el origen de los productos o servicios (o dicho de otro modo, la confianza que se tenga en ellos) da valor al producto mismo. Este análisis debe incluir, por cierto, tanto el rol del Estado como el de los privados.

\section{DIPLOMACIA PÚBLICA Y PODER BLANDO}

Desde la perspectiva de las relaciones internacionales, en las últimas décadas se han difundido las ideas de Diplomacia Pública (Public Diplomacy) y Poder Blando (Soft Power) como nuevas estrategias de persuasión por parte del Estado. Éstas incluyen mecanismos de comunicación por parte de las naciones en el escenario internacional, donde las herramientas tradicionales del «poder duro» (primordialmente basadas en el poderío militar) y la diplomacia ya no son suficientes en un nuevo escenario crecientemente complejo: «Estamos entrando en una nueva era en la cual el conocimiento, la cultura y las comunicaciones son la clave, no sólo para el progreso tecnológico y la prosperidad económica sino que también para la cohesión social y el desarrollo sustentable» (Potter, 2002, p.1).

En sus orígenes, el término Diplomacia Pública fue definido por la Agencia de Informaciones de Estados Unidos a principios de la década de los sesenta como:

\begin{abstract}
«(..) la influencia de actitudes públicas en la formación y ejecución de la política exterior. Comprende dimensiones de las relaciones internacionales, más alla de la diplomacia tradicional; el cultivo por parte de los gobiernos de la opinión pública de otros países; la interacción de grupos privados de interés con sus pares en otros países; el reporte de los asuntos internacionales y su impacto en la política exterior; la comunicación entre aquellos cuya labor es la comunicación como es el caso de los diplomáticos y corresponsales extranjeros; y los procesos de comunicación intercul tural» (en Anholt, 2007, p.12)
\end{abstract}

En su versión original entonces, el concepto de diplomacia pública estaba dirigido no sólo a la relación di-
7 Sin embargo, algunos organismos internacionales han empezado a abordar

el tema de la confianza como una prioridad en el escenario mundial. En su reporte anual del año 2002-2003, el Foro Económico Mundial dio cuenta de la necesidad de poner en la agenda pública el tema de la confianza, focalizándose en «temas fundamentales de confianza, valores, seguridad y geopolítica; gobierno global, perspectivas de la economía global y desafíos corporativos» (Building Trust, Peace and Reconciliation, 2002/2003 p.4).

8 De acuerdo al modelo desarrollado por el Reputation Institute (www. reputationinstitute.com), la Reputación Corporativa posee siete dimensiones: Productos y Servicios, Innovación, Ambiente de Trabajo, Gobierno Corporativo, Ciudadanía Liderazgo y Desempeño Financiero. 
9 La tesis de Nye se basa en la realidad norteamericana y radica en que las

tradicionales fuentes de persuasión de la Guerra Fría, esto es persuasión basada en el poderío militar

o hard power está dando

paso a un poder más difuso.

Actualmente, sin embargo,

la discusión se ha centrado

en analizar la creciente

influencia de China en el

mundo occidental y cómo a

través de su poder blando ha logrado influenciar diversos ámbitos de mundo occidental (Ramo \& Foreign Policy Centre 2004: Saunders, 2006). Para la realidad chilena es interesante leer el

artículo «La expansión del Soft Power en Chile: Una oportunidad de Desarrollo» (Rovira, 2006).

10 En español, y particularmente en Chile, este concepto se le ha denominado Imagen País.

11 Según el propio Anholt: «El Índice Anholt de Marcas País, fundado en 2005 es un catastro trimestra que recoge, mide y analiza las percepciones de unos 26.000 ciudadanos de 35 países sobre un total de 30 a 40 países» (Anholt, 2008b,

pp.1). El Índice Anholt de Marcas Ciudad efectúa un análisis similar de 40-60 ciudades.

\section{Una corbata italiana, un vino francés o productos con tecnología japonesa tienen un prestigio ganado entre los consumidores del mundo y, por lo tanto, pueden agregar este valor al precio final del producto.}

plomática entre Estados, sino que también afectaba a la esfera privada y la opinión pública en el extranjero, lo que significó ampliar la visión de la diplomacia tradicional. En palabras más simples, tomadas de Hans Tuch, es «el proceso de comunicación hacia públicos extranjeros, ejercido por un gobierno, cuyo objetivo es generar un entendimiento de las ideas e ideales de la nación, sus instituciones y cultura, así como de sus objetivos nacionales y políticas actuales» (Potter, 2002, p.3).

Haciendo un paralelo con la empresa privada, la diplomacia pública contiene en su esencia los conceptos propios de la disciplina de las relaciones públicas, aunque esta vez, desde los estamentos de una nación, hacia sus públicos externos, lo que en términos genéricos se refiere a la comunidad internacional o bien a determinados actores dentro de ésta. En términos habermasianos, algo así como un «espacio público» Internacional (Habermas, 1984)

El concepto de poder blando (soft power), creado a principios de la década de los noventa por el norteamericano Jospeh Nye, contiene algunas ideas de la diplomacia pública. Sin embargo, su perspectiva tiene como base las relaciones internacionales, con fuerte acento en la idea de seguridad nacional. Esta última, no obstante, desde una visión más amplia, donde no sólo la fuerza militar es primordial sino que también «las comunicaciones, las habilidades organizacionales e institucionales y la manipulación de la interdependencia» (J. Nye, 1990 , p. 158).

En palabras del propio autor, se refiere a «en qué medida un determinado Estado [específicamente individualizado en el caso de los Estados Unidos] es capaz de controlar el ambiente político y lograr que otros países hagan lo que este quiera» (J. Nye,1990., p. 155). En otras palabras, el poder del Estado radica en la capacidad de persuasión que tenga frente a otros estados ${ }^{9}$, proviniendo ésta del atractivo de su cultura, instituciones políticas (J. S. Nye, 2004), lo que implica un paso adelante pues interrelaciona los procesos de persuasión con la identidad de las naciones.

Sin embargo, tanto el concepto de Diplomacia Pública como el de Poder Blando se remiten principalmente al ámbito político y, aunque en la práctica ambos repercuten en el área económica, sólo lo hacen en forma indirecta. Asimismo, ambos conceptos delimitan su análisis al rol del Estado en la gestión de la persuasión, sin involucrar directamente a los entes privados.

\section{LA TEORÍA DE COMPETITIVE IDENTITYO NATION BRANDING}

En los años noventa, el británico Simon Anholt desarrolló el concepto de Nation (Place) Branding, traducido al español como «marca país» ${ }^{10}$, el que posteriormente evolucionó a la idea de Competitive Identity. Anholt es también el creador del primer indicador de «branding» aplicado a países, el índice Anholt de Marcas País. ${ }^{11}$

En esencia, la teoría apuesta a unir los conceptos de valor del origen del producto (esto es, el país o ciudad donde el producto o servicio se origina), junto al valor de la marca corporativa, los que deben actuar con sinergia puesto que un «amplio rango de productos de consumo proviene de un aún más amplio abanico de países, lo que aumenta la necesidad de construir confianza tanto desde la compañía como del país de origen» (Anholt, 2007, p. 20).

Así, una corbata italiana, un vino francés o productos con tecnología japonesa tienen un prestigio ganado entre los consumidores del mundo y, por lo tanto, pueden agregar este valor al precio final del producto, ya 
que han conseguido diferenciarse y construir una reputación propia que da confianza al consumidor.

Esta teoría, si bien utiliza como base conceptos tradicionales del marketing y del branding aplicados a la gestión de imagen de ciudades, regiones y países (Kotler \& Gertner, 2001), también toma elementos extraídos de las ideas provenientes de la Diplomacia Pública y el Poder Blando, sintetizando teorías de origen público y privado (Anholt, 2007). De hecho, si se analizan los seis «canales naturales» por los que según Anholt (Anholt, 2007 , p. 25-26) se transmite la reputación de los países $^{12}$, se aprecia una combinación de aspectos públicoprivados que actúan sobre la imagen del país como un todo, desmitificándose la idea de que la construcción de una marca país se basa sólo en el diseño de un eslogan, un logo poderoso y un importante gasto en campañas de marketing (Anholt, 2008b) por parte del gobierno. Más bien, desde la identidad de cada país se crea la imagen, distinguiendo, al igual que otros estudiosos de la comunicación (Tironi \& Cavallo, 2004) entre ambos conceptos. En el ya mencionado Reputation Institute, los factores que determinan la marca país también van estrechamente ligados con la identidad del territorio e incluyen factores tan diversos como las variantes climáticas, las marcas asociadas, la apertura comercial al exterior, el marco político y la coyuntura internacional, entre otros (Prado, 2007) $)^{13}$

De esta manera, para un país como Chile, su imagen en el extranjero dependería no sólo del buen manejo de las comunicaciones externas o de las políticas macroeconómicas del gobierno, sino que igualmente de comportamientos particulares de nuestros líderes (por ejemplo, el caso de futbolistas nacionales que han dejado su impronta en el mundo europeo), así como también por el reconocido valor de marcas tradicionales del vino nacional. De la misma forma, la mala experiencia de un turista en nuestro territorio actuaría en detrimento de la imagen global.

Sin embargo, y a pesar de todos los factores que pudieran afectar o construir la imagen país, la comunicación de ésta debe tener como objetivo crear una idea simple, clara y diferenciadora, basada en características emocionales para que sean entendidas por todas las audiencias y en distintas situaciones (Jaffe \& Nebenzahl, 2001).
En las últimas décadas, diversas economías emergentes han intentado implementar este concepto de marca país, con diferentes resultados, dependiendo de la puesta en práctica de estos postulados teóricos Asimismo, diversas críticas han surgido respecto de la factibilidad de que una estrategia de marca país sea la mejor manera de contribuir al desarrollo económico (Fan, 2006)

Entre las experiencias exitosas se encuentran Nueva Zelandia con «New Zealand 100\% Pure» (www.newzealand.com) y Sudáfrica (www.brandsouthafrica.com), quienes han logrado transmitir en sus campañas (lideradas por el Estado pero en estrecha colaboración con el mundo privado) la identidad propia del lugar y comunicarlo exitosamente. En Latinoamérica, destacan las iniciativas de Colombia (www.colombiaespasion.com) y, en menor medida, Perú. La experiencia de Argentina, con su lema «Argentina, un país en serio», muestra cómo un eslogan no sobrevive si no existe un acuerdo entre la identidad y la imagen, y si no es, además, producto de un consenso en el país ${ }^{14}$.

En Chile, la iniciativa de crear una imagen país se materializó por primera vez en 2004, fundamentando esta decisión en:

«La importancia para el desarrollo económico del país de las exportaciones de bienes y servicios incluido el turismo, así como la atracción de la inversión extranjera; las oportunidades que representan para el país los recientes Tratados de Libre Comercio con importantes países y bloques regionales, así como los que se están negociando; la importante cantidad de recursos invertidos en su posicionamiento por otros países que compiten con Chile en exportaciones, turismo y atrac ción de inversiones; la necesidad expresada por diversos sectores económicos del país de contar con un respaldo que potencie lo invertido en la construcción de la imagen de sus productos, procesos y servicios en el exterior, así como los esfuerzos en la captación de inversiones» (Direcon, 2004).

Sin embargo, a cuatro años de la puesta en marcha de la idea «Chile, All Ways Surprising», «Chile, Sorprende Siempre», es posible apreciar algunas señales de que la gestión de marca país en Chile se está replanteando. En primer lugar, se ha creado una nueva institucionalidad, que además es independiente un ministerio en particu-
12 De acuerdo a Anholt, los seis canales por los cuales se comunica un país con sus audiencias - ya sea de manera planificada o accidentalson: promoción turística, marcas de exportación. políticas de gobierno Idomésticas u orientadas al exterior), la manera en que se solicita inversión o se realiza la atracción de «talentos», intercambio cultural y comportamiento de los líderes del país lya sean éstos políticos 0 deportistas).

13 Según esta entidad los factores que determinan la imagen de un país son: Variantes climáticas; Productos y servicios: Empresas y corporaciones de productos y servicios; Instituciones de la sociedad civil; Sectores económicos; Especialización productiva; Grado de internacionalización de la economía; Posicionamiento económico de riesgo/ país; Marco geopolítico: Relaciones coyunturales internacionales; Aspectos antropológicos, culturales, artísticos, literarios, monumentales, festivos, folklóricos, gastronómicos, etc. e Historia y estructura social (Prado, 2007). 
14 Esta idea partió desde la campaña de Néstor Kirchner a la Casa Rosada y luego se aplicó como base central de la Imagen País de Argentina. Sin embargo, no sobrevivió a las críticas de la oposición quiénes ironizaban respecto de la lejanía entre el eslogan y la realidad

del país, tanto por algunos episodios a nivel local

como el incumplimiento de compromisos a nivel internacional. Actualmente se prepara un relanzamiento de la marca país de

Argentina para 2010 con el fin de conmemorar el Bicentenario de la Revolución de Mayo.

15 Hasta el anuncio de la Presidenta Michelle

Bachelet en el discurso presidencial del 21 de Mayo, la gestión de la Marca País se realizaba bajo la dirección de Pro Chile y, en menor medida, de CORFO.

16 La campaña «Chile, All Ways Surprising» fue realizada por Interbrand Actualmente, es la multinacional Ogilvy la que se encuentra a cargo del tema. lar. Así, la tarea de afrontar la temática de marca país ha sido encomendada a un nuevo equipo conocido como Proyecto Imagen País, que actualmente depende de la Presidencia de la República, pero que pretende contar con una personalidad jurídica propia de carácter privado. De este modo, se produce una desvinculación del accionar particular de una repartición pública ${ }^{15}$, lo que le confiere una visión más global y estratégica que, a su vez, demuestra la relevancia e independencia que se desea dar al tema. Además, la idea de constituirse como una fundación de carácter privado le permitirá contar con una mayor agilidad e independencia en la toma de decisiones.

Un segundo elemento lo constituye el aumento de fondos en el presupuesto nacional: durante el discurso presidencial del 21 de Mayo se informó que la iniciativa contará con una inyección de 40 millones de dólares para el presupuesto 2009, lo que permitirá su puesta en marcha. Otra señal es la renovación que se ha llevado a cabo en las labores creativas. Luego de licitación públi$\mathrm{ca}$, se ha escogido una nueva agencia publicitaria ${ }^{16}$ que estará a cargo de la creación estratégica. En esa línea, un factor importante es la contratación del británico Simon Anholt, quien asesorará directamente el proyecto de Imagen País. Junto a ello, y desde el próximo año, Chile será parte del ranking Anholt-GfK Roper Nations Brand Index, que trimestralmente es publicado en conjunto por Anholt y la consultora GfK Roper, lo que permitirá contar con un elemento de medición empírica que pueda efectivamente evaluar el posicionamiento de la marca Chile a nivel mundial y comparar su evolución en el tiempo.

Estos cambios ponen en relevancia no sólo la necesidad de trabajar el tema estratégicamente y en cooperación público-privada, sino que también desde la propia identidad de la nación como elemento clave para propiciar el desarrollo económico.

\section{CONCLUSIONES}

Este artículo ha pretendido sentar algunas bases teóricas sobre la importancia de generar un enfoque más amplio e interdisciplinario en el tema del desarro- llo económico y, por ende, de la necesidad de incorporar nuevos elementos al análisis, tal como la creación de confianza. Mediante una exploración desde distintas disciplinas (relaciones internacionales, sociología, marketing y comunicación) se ha intentado establecer la idea de que el desarrollo económico es un tema complejo y multicausal, y que tanto las teorías como las políticas deben entenderse desde una perspectiva que incluya distintas áreas del conocimiento.

Este enfoque, sin embargo, no está exento de problemas. Existen dificultades de medición de conceptos como cultura, confianza o reputación a la hora de realizar correlaciones estadísticas con el desarrollo económico. Éstas están siendo encaradas por las ciencias sociales, mientras diversos académicos e instituciones se encuentran en la búsqueda de nuevas metodologías que permitan desarrollar más y mejores trabajos empíricos.

En este artículo seminal se ha intentado poner en relevancia aspectos que, si bien no constituyen una única respuesta al camino del crecimiento, presentan nuevas alternativas complementarias a la visión tradicional del desarrollo económico. Una de las novedades de utilizar conceptos como confianza, reputación o analizar la teoría de Competitive Identity es justamente dar un paso adelante en el tema de la dicotomía Estado-Mercado, ya que ninguno de éstos conceptos depende de uno u otro actor, sino que más bien de la acción articulada y sinérgica entre ambos.

En el caso de Chile, un país en vías de desarrollo muy inserto en la economía internacional, este tipo de variables se torna especialmente relevante. Chile ha escogido liberalizar su economía a través de una política de bajos aranceles y de acuerdos comerciales bilaterales con las principales zonas económicas del mundo. Sin embargo, la firma de tratados comerciales que permitan agilizar el intercambio económico con el exterior es sólo el inicio. Es necesario agregar valor de origen a nuestras diversas ofertas, a la vez que posicionar al país como polo atractivo para las inversiones y el turismo. En otras palabras, constituirse en una alternativa verdaderamente confiable. 


\section{REFERENCIAS}

Anholt, S. (2007). Competitive Identity: The Brand Management for Nations, Cities and Regions. Basingstoke: Palgrave Macmillan.

Anholt, S. (2008a). Editorial. Place Branding and Public Diplomacy 4(2).

Anholt, S. (2008b). Las Marcas Pais. Paper presented at the Seminario Internacional Imagen País, 2008, Santiago de Chile.

Axelrod, R. (1984). The Evolution of Cooperation New York: Basic Book Publishers

Bhagwati, J. N. (2004). In Defense of Globalization. New York: Oxford University Press.

Bourdieu, P. (2005). The Social Structures of the Economy. Oxford: Polity Press.

Building Trust, Peace and Reconciliation. (2002/2003): World Economic Forum.

Child, J. (1998). Trust and International Strategic Alliances: The case of Sino - Foreign Ventures. In C. Lane (Ed.), Trust Within and Between Organizations (pp. 241272). Oxford: Oxford University Press.

Coleman, J. S. (1988). Social Capital in the Creation of Human Capital. The American Journal of Sociology, 94 Supplement: Organizations and Institutions: Sociological and Economic Approaches to the Analysis of Social Structure, pp. 95-120.

Dasgupta, P., \& Serageldin, I. (2000). Social Capital: A Multifaceted Perspective. Washington D.C.: World Bank.

Dicken, P. (2003). Global Shift: Reshaping the Global Economic Map in the 21st century (4th ed.). London: Sage.

Direcon, D. d. C. P. C.-. (2004). Comunicado de Prensa: Gobierno, sector público y empresa Interbrand se compromenten con la imagen de Chile en el exterior. (pp. 2).

Fan, Y. (2006). Branding the Nation: What is being branded? Journal of Vacation Marketing, 12(1), pp. 5-14.

Friedman, T. L. (2006). The World is Flat: The Globalized World in the Twenty-first Century. London: Penguin.

Fukuyama, F. (1995). Trust: The Social Virtues and the Creation of Prosperity. London: Hamish Hamilton.
Gambetta, D. (1988). Trust: making and breaking cooperative relations. Oxford: Basil Blackwell.

Gilpin, R., \& Gilpin, J. M. (2001). Global Political Economy: Understanding the International Economic Order. Princeton, N.J.: Princeton University Press.

Glaeser, E. L., David and Sacerdote, Bruce (2002). An economic Approach to Social Capital. The Economic Journal, 112, pp. 437-458.

Granovetter, M. (1985). Economic Action and Social Structure: The Problem of embeddedness. The American Journal of Sociology, 91(3), pp. 481-510.

Guardia, A. (2008). Comercio Exterior de Chile. Cuarto Trimestre 2007. In G. d. C. Direccion de Relaciones Economicas Internacionales - Prochile (Ed.).

Habermas, J. (1984). The Theory of Communicative Action. Boston: Beacon Press.

Hardin, R. (2002). Trust and Trustworthiness. New York: Russell Sage Foundation.

Hardy, C., Phillips, N., \& Lawrence, T. (1998b). Distinguishing Trust and Power in Interorganizational Relations: Forms and Facades of Trust. In C. Lane (Ed.), Trust within and between organizations (pp. 64 - 87). Oxford: Oxford University Press.

Harrison, L. E. (1992). Who Prospers?: How Cultural Values Shape Economic and Political Success: New York: Basic Books.

Held, D. (1999). Global Transformations: Politics, Economics and Culture. Oxford: Polity Press.

Humpfrey, J. (1998). Trust and the transformation of Supplier Relations in Indian Industry. In C. Lane (Ed.), Trust Within and Between Organizations (pp. 214-240). Oxford: Oxford University Press.

Huntington, S. P., \& Harrison, L. E. (2000). Culture Matters: How Values Shape Human Progress. New York: Ba sic Books.

Inglehart, R. (2004). Human Beliefs and Values: a crosscultural sourcebook based on the 1999-2002 values surveys. Mexico.

Jaffe, E. D., \& Nebenzahl, I.D. (2001). National image and competitive advantage: the theory and practice of country-of-origin effect. Copenhagen: Copenhagen Business School Press; Abingdon: Marston. 
Knack, S., \& Zak, P. J. (2001). Trust and Growth. The Economic Journal, 111(470), 295-321.

Knight, J. (2001). Social Norms and the rule of Law: Fostering Trust in a Socially Diverse Society In K. S. Cook (Ed.), Trust in society (Vol. II, pp. 354-373). New York: Russell Sage Foundation.

Kotler, P., \& Gertner, D. (2001). Country as a brand, product, and beyond: A place marketing and brand management perspective. Brand Management, 9(4-5), pp.219-261.

Luhmann, N. (1979). Trust and Power: Two Works. Chichester: Wiley.

McKnight, Harrison, D., \& Chervany, N. L. (2006). Reflections on an initial trust-building model. In R. Bachmann \& A. Zaheer (Eds.), Handbook of Trust Research (pp. 29-51). Cheltenham: Edward Elgar.

Nye, J. (1990, Fall 1990). Soft Power. Foreign Policy, pp. 153-171.

Nye,J. S. (2004). Soft Power: The Means to Success in World Politics. New York: Public Affairs.

Ohmae, K. (1996). The End of The Nation State: The Rise of Regional Economies (Special overseas ed.). London: HarperCollins.

Porter, M. (2000). Atitudes, Values, Beliefs and the Micro economics of prosperity. In S. P. Huntington \& L. E. Harrison (Eds.), Culture matters: how values shape human progress (pp. 2-13). New York: Basic Books.

Potter, E. H. (2002). Canada and the New Public Diplomacy Clingendael Discussion Discussion Papers in Diplomacy, Netherlands Institute of International Relations.

Prado, F. (2007). Marcas, Reputaciones y Territorios, from http://reputationinstitute.com/events/Marcas_ Reputaciones_y_Territorios_Badajoz2007.pdf

Putnam, R. D. (1993). Making Democracy Work: Civic Traditions in Modern Italy. Princeton, N.J.: Princeton University Press.

Putnam, R. D. (2000). Bowling Alone: The Collapse and Revival of American Community. New York: Simon \& Schuster.
Putnam, R. D. (2007). E Pluribus Unum: Diversity and Community in the Twenty-first Century. The 2006 Johan Skytte Prize Lecture

Scandinavian Political Studies, 30(2), pp.137-174.

Ramo, J. C., \& Foreign Policy Centre (London England) (2004). The Beijing consensus. London: Foreign Policy Centre

Rodrik, D. (2002). After Neo- liberalism, what?, Alternatives to Neo Liberalism Conference. Washington DC.

Rovira, C. (2006). La expansión del Soft Power en Chile: Una oportunidad de Desarrollo. Santiago de Chile: Asuntos Públicos.

Said, E. (2002). The Clash of Definitions. In H. Y. Jung (Ed.), Comparative Political Culture in the Age of Globalization: An Introductory Anthology (pp. 363-380). Lanham, Md.; Oxford: Lexington Books.

Saunders, S. D. a. R. A. (2006). Talking Up China: An Analysis of China's Rising Cultural Power and Global Promotion of the Chinese Language. East Asia, 23(2).

Schech, S., \& Haggis, J. (2000). Culture and Development: A Critical Introduction. Oxford; Malden, MA: Blackwell Publishers.

Sen, A. K. (1999). Development as Freedom. New York: Knopf.

Sztompka, P. (1999). Trust: A Sociological Theory. Cambridge: Cambridge University Press.

Thomas, R. M. (1999). Human Development Theories: Windows on Culture. Thousand Oaks, Calif.; London: Sage Publications.

Throsby, C. D. (2001). Economics and Culture. Cambridge: Cambridge University Press.

Tironi, E., \& Cavallo, A. (2004). Comunicación Estratégica: Vivir en un mundo de señales. Santiago: Taurus.

Zucker, L. G. (1986). Production of Trust: Institutional Sources of Economic Structure, 1840 - 1920 Research in Organizational Behavior, 8, 53 -111. 


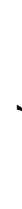

